\title{
Saikosaponin-d suppresses cell growth in renal cell carcinoma through EGFR/p38 signaling pathway
}

\author{
C. CAI ${ }^{\ddagger}$, H. ZHANG ${ }^{\ddagger}$, Y. OU $U^{\ddagger}$ Y. JIANG, D. ZHONG, H. QI*, Q. DANG* \\ Department of Urology, Nanfang Hospital, Southern Medical University, Guangzhou, Guangdong 510515, P.R. China \\ *Correspondence: 2668241842@qq.com, dangqiang0128@163.com \\ ${ }^{*}$ Contributed equally to this work.
}

Received June 20, 2016 / Accepted January 17, 2017

\begin{abstract}
The study aimed to explore the effect of Saikosaponin-d (SSd) and its underlying mechanism on cell growth inhibition as well as induction of apoptosis and cell cycle arrest in renal cell carcinoma (RCC). MTT assay and colony formation assay were employed in this study, with the results indicating that RCC cells proliferation was inhibited by SSd at different doses. Analysis by flow cytometry revealed that RCC cell proliferation inhibitory effect of SSd was achieved by inducing apoptosis and cell cycle arrest at G0/G1 phase via up-regulation of p53. As compared to the control group, SSd can significantly inhibit the growth of 769-P and 786-O cell lines and induce apoptosis and cell cycle arrest. The mechanism exploration demonstrated that inhibiting the activation of EGFR/p38 signaling pathways was the molecular basis of SSd's biological effects such as inducing apoptotic death, inhibiting cell growth as well as up-regulating p53 expression in human RCC cells. In conclusion, our data suggest that SSd may serve as a promising intervention for chemopreventive or chemotherapeutic treatment for patients with RCC.
\end{abstract}

Key words: Saikosaponin-d, RCC, cell growth, EGFR/p38

As one of the world's most common malignant tumor, Renal cell cancer (RCC) contributes about 2 3\% of the malignancies in adult population, with an increase of $2 \sim 3 \%$ every decade in terms of its morbidity $[1,2]$. The major treatments for RCC include partial nephrectomy ( $<4 \mathrm{~cm}$ in the largest dimension) and nephrectomy ( $>4 \mathrm{~cm}$ in the largest dimension) if, of course, the patients are "lucky enough" to be detected and diagnosed in the early stage. Nevertheless, postoperative recurrence or metastasis, which accounts for about $30 \sim 40 \%$ of the total cases, is still a major concern for these patients, leading to at least a hundred thousand annual deaths [3, 4]. Medical interventions available for RCC patients with metastasis are chemotherapy, radiotherapy, and immunotherapy and so on. However, the effect has been proved being limited, creating an urgent demand for a new solution with great safety and efficacy in RCC treatment.

Saikosaponin-d (SSd) is an extract from Bupleurum falcatum L, usually used as a common drug material in traditional Chinese medicine (TCM) for anti-inflammatory and antiinfectious purposes $[5,6]$. According to some reports, this saponin derivative was found to have some pharmacological activities similar to sterides such as pain-relieving, antiinflammation, immunoregulation, anti-viral function, and hepatocyte protection [7]. Its anti-neoplastic effect, however, was reported until recently by the studies that focused on the fields of thyroid cancer [8], human non-small cell lung cancer (NSCLC) [9] and liver cancer [10]. The action mechanism and targets or pathways of SSd are still unclear and subject to further exploration.

Being a member of the ErbB receptor tyrosine kinase family, epidermal growth factor receptor (EGFR) serves as an essential factor in multiple cellular processes involving with cell proliferation, survival, and cell migration [11-13]. The over-expression of EGFR has been reported in cases with clear-cell RCC tumors in recent studies and the association between EGFR expression and cancer progression has been confirmed [14-16]. According to the pre-clinical study of Ming Feng Chena, SSd is found to carry a proliferation and migration inhibiting function in HSC-T6 cells in rat models via the pathway that regulates the phosphorylation of $\mathrm{p} 38$ and ERK1/2 [17]. In this study, we attempted to look into the effect of SSd on cell proliferation and apoptosis through 
EGFR/p38 pathway in RCC, which was the first try in related research areas.

\section{Materials and methods}

Human RCC cell lines (769-P and 786-O) provided by Medical School of Xi'an Jiaotong University were employed for cell culture. Buffer used to preserve the cells was prepared with RPMI-1640 containing 10\% fetal bovine serum (FBS), $50 \mu \mathrm{g} / \mathrm{ml}$ streptomycin, $100 \mathrm{IU} / \mathrm{ml}$ penicillin and $2 \mathrm{mM}$ of Lglutamine. The cells were cultured at $37^{\circ} \mathrm{C}$ under a humidified condition with $5 \% \mathrm{CO}_{2}$.

Chemicals. SSd purchased from Shfeng (Shanghai, China) was dissolved in $50 \mathrm{mM}$ of DMSO and then preserved at $-20^{\circ} \mathrm{C}$ for further use; anti-human p53, c-Fos, $\beta$-Actin and GAPDH antibodies were obtained from Santa Cruz Biotechnology; and Cell Signaling Technology was the provider for antibodies against EGFR, p-EGFR, p-ERK, MEK, p-MEK, p38 and p-p38; Recombinant Human EGF, AG1478 and SB203580 were purchased from Peprotech, Selleckchem and Medchem Express, respectively; Nanjing KeyGen Biotech was the manufacturer for Annexin V-FITC Apoptosis Assay kit and Cell Cycle Assay Kit.

MTT assay (a tetrazolium-based assay) was employed for cell viability determination using 96-well plates, with $50 \mu \mathrm{l}$ of media which contains one thousand cells in each well. The cells were treated respectively with different doses of SSd or with buffer not containing SSd ( $0 \mu \mathrm{M}$ of SSd) serving as the control group before incubation for several times; after the addition of $0.5 \mathrm{mg} / \mathrm{ml}$ of MTT, the cells were incubated at $37^{\circ} \mathrm{C}$ for another hour; the supernatant was removed and the cells were dissolved with DMSO; finally, a 96-well microplate reader was used for colorimetric analysis at $490 \mathrm{~nm}$ [18]. The procedure was carried out in triplicate.

Colony formation. To determine the inhibition effect of SSd on colony formation in RCC cells, two different types of RCC cells-769-P and 786-O RCC cells were respectively seeded in 24-well plates ( 100 cells/well), and 10, 20 and $30 \mu \mathrm{M}$ of SSd was added respectively into the wells for a 14-day culture. Then the cells were stained using crystal violet for colony counting.

DNA cell cycle. In the cell cycle test, the cells were treated respectively with 0 and $20 \mu \mathrm{M}$ of SSd for $48 \mathrm{~h}$ when a $60-80 \%$ confluence was obtained; the cells were washed twice with PBS before fixation with $70 \%$ ethanol at $4^{\circ} \mathrm{C}$ for $1 \mathrm{~h}$; after another wash with PBS, $0.05 \mathrm{mg} / \mathrm{ml}$ RNase-contained propidium iodide (PI) solution was added to re-suspend the cells, which were then incubated for $30 \mathrm{~min}$ at room temperature avoiding light for DNA content analysis using flow cytometer.

Apoptosis. During the procedure of apoptosis quantitative determination, the 769-P and 786-O cells were processed respectively with 10 and $20 \mu \mathrm{M}$ of SSd for $48 \mathrm{~h}$. Annexin VFITC Apoptosis Assay kit was employed for Annexin V and PI staining according to the operative instruction from the kit provider. And then flow cytometry was used for apoptotic cell analysis.
Western blot. When it came to western blot analysis, RIPA buffer was prepared using Tris- $\mathrm{HCl}(50 \mathrm{mM}, \mathrm{pH} 7.4), \mathrm{NaCl}$ $(150 \mathrm{mM})$, EDTA (1 mM), 1\% NP-40, PMSF (1 mM), NaF (1 mM), $\mathrm{Na}_{3} \mathrm{VO}_{4}(1 \mathrm{mM})$, okadaic acid $(1 \mathrm{mM})$, as well as 1 $\mathrm{mg} / \mathrm{ml}$ pepstatin, aprotinin and leupeptin; the cells were lysed in RIPA buffer and Protease Inhibitor Cocktail (provided by Roche Inc.); $20 \mu \mathrm{g}$ of protein was sampled, with $10-15 \%$ gel prepared for SDS-PAGE; the protein was transferred onto PVDF membranes (provided by Millipore) after electrophoresis procedure, followed by membrane blocking at room temperature for $1 \mathrm{~h}$ using 5\% BSA diluted in PBS; then the membranes, after processed with diluted primary antibody solutions, were incubated overnight at $4^{\circ} \mathrm{C}$; after another wash, anti-rabbit, anti-mouse or anti-goat IgG HRPs was used as the secondary antibody to treat the membranes before they were incubated for another hour; in the last step, ECL mixture (provided by Thermo Fisher Scientific) was employed for color developing for the blots[19].

Statistical analyses. In this study, SPSS 22.0 was used for all the statistical analyses. Quantitative data were presented as mean \pm SE and one-way ANOVA was employed for intergroup comparisons. If statistical difference was shown, then Dunnett's t-test was established for further comparison between paired groups. Student's t-test was also used when only 2 groups were involved in statistical comparison. $\mathrm{P}<0.05$ was considered to represent a significant statistical difference.

\section{Results}

Figure 1 shows the growth inhibitory effect of SSd (Figure $1 \mathrm{~A}$ - chemical structure) on RCC cells by comparing the cell viability between groups treated with different doses of SSd as well as between different time points of the groups treated with the same dose of SSd. The results revealed that SSd presented a proliferation inhibitory activity in 769-P and $786-\mathrm{O}$ cells. The viability rate of RCC cells was $67 \%$ (10 $\mu \mathrm{M}$ group), $34 \%$ (15 $\mu \mathrm{M}$ group) and $15 \%$ (20 $\mu \mathrm{M}$ group) respectively through a 48 -hour SSd treatment (Figure 1B). Also, an declining viability rate was found at the time points of 24,48 and $72 \mathrm{~h}$ in the groups treated with $15 \mu \mathrm{M}$ of SSd (Figure 1C), indicating a dose- and time-dependent inhibitory effect on RCC cells.

For colony formation assay, 769-P and 786-O cells were respectively seeded into 24 -well plates, 100 cells per well, and cultured for 14 days after SSd treatment $(10,20$ and $30 \mu \mathrm{M}$, respectively). As shown in Figure 2, the colony counting was remarkably decreased with the increase of SSd dose, indicating that SSd can significantly inhibit colony formation in both of 769-P (Fig. 2B) and 786-O (Figure 2C) cells.

To understand how SSd influences cell cycle in RCC cells, flow cytometry was employed to determine the percentage of the cells at different phases after a 24-hour SSd treatment process $(20 \mu \mathrm{M})$. The results of RCC cell cycle assay showed a significantly elevated percentage level of G0/G1 phase cells and an obviously declined level in $\mathrm{S}$ phase cells compared with 
the control group $(0 \mu \mathrm{M})$ (Figure 3$)$, revealing that SSd has the ability to induce cell cycle arrest by inhibiting cell growth from $\mathrm{G} 0 / \mathrm{G} 1$ phase.

The effect of SSd on RCC cell apoptosis was measured by Annexin $\mathrm{V}$ positive cells and western blot analysis after 769-P and 786-O cells were respectively treated with 10 and $20 \mu \mathrm{M}$ of SSd for 48 hours. According to the flow cytometry data (Figuer 4A), Annexin $\mathrm{V}$ positive cells increased after SSd treatment, and compared to the control group $(0 \mu \mathrm{M})$, significant differences were found in SSd treatment groups, where the percentage of Annexin V positive cells was re-
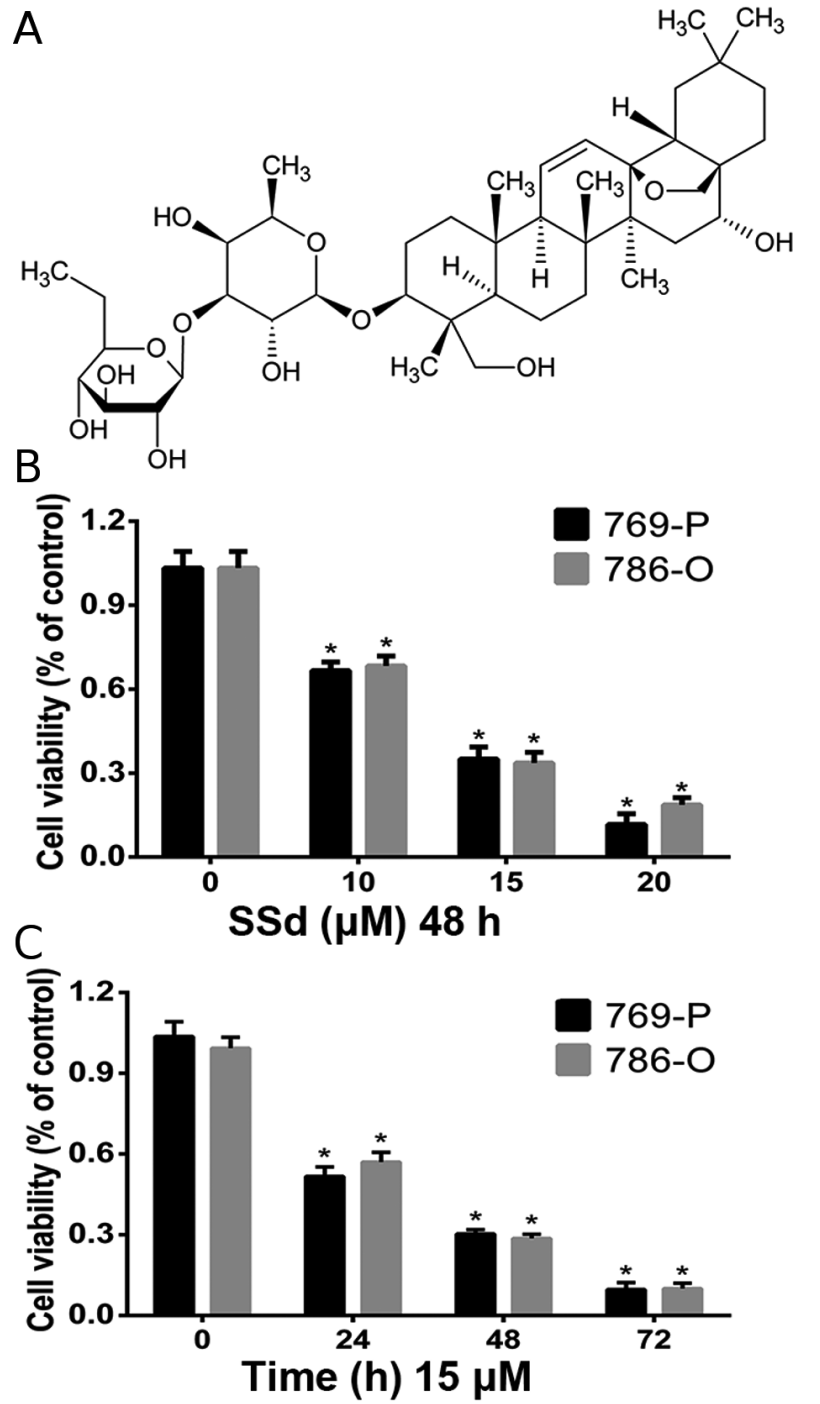

Figure 1. The growth inhibitory effect of SSd on RCC cells. (A) The chemical structure of SSd. (B) Cell viability was determined after RCC (769-P and786-O) cells were treated respectively with different doses of SSd (10, 15 and $20 \mu \mathrm{M}$ ) for $48 \mathrm{~h}$. (C) Cell viability was determined at different time points $(0,24,48$, and $72 \mathrm{~h})$ after RCC $(769-\mathrm{P}$ and $786-\mathrm{O})$ cells were treated with $15 \mu \mathrm{M}$ of SSd. MTT assay was employed to determine the cell viability. ${ }^{\star} \mathbf{P}<0.05$ represents a statistically significant difference. spectively $9.6 \%(10 \mu \mathrm{M}$ group) and $16.6 \%(20 \mu \mathrm{M}$ group $)$ in $769-\mathrm{P}$ cells, and $21.2 \%(10 \mu \mathrm{M}$ group) and $49.8 \%(20 \mu \mathrm{M}$ group) in 786-O cells (Figure 4B). The western blot analysis demonstrated an increase in p53 after treatment with SSd (Figure 4C). These results indicate SSd has apoptosis inducing effect on RCC cells.

In this study, the mechanism of SSd on its cell proliferation inhibitory effect was also investigated. Further study on the expression of cell growth related genes suggested that SSd can significantly inhibit the activation of the EGFR pathway, as EGFR, phospho-EGFR, MEK, phospho-MEK, p38, phosphop38 and its downstream c-Fos all showed a decrease after SSd treatment (Figure 5A, 5B). The expression of ERK, however, presented an increase tendency, which was considered to be related to its activity on (endoplasmic reticulum) stress induction.

As EGFR is known to regulate cell proliferation via the MAPK pathway [20], our exploration was mainly focused on verification of the hypothesis that EGFR/MAPK signaling pathway is the one through which SSd implements its regulatory function on RCC cell growth. EGFR inhibitor AG1478 [21] and p38 inhibitor SB203580 [22] were used as positive controls, and an inhibitory effect similar to these inhibitors was found in SSd, characterized by blocking EFG induced pEGFR and activation of p38 (Figure 5C, 5D), which indicates that SSd can block the EGFR/p38 signaling pathway. Then MTT assay was employed to detect cell viability of 769-P and 786-O groups after treatment with SSd ( $2 \mathrm{~h}$ ) and EGF ( $48 \mathrm{~h}$ ), where EGFR inhibitor AG1478 or p38 inhibitor SB203580 was also used as positive control (Figure $5 \mathrm{E}, 5 \mathrm{~F}$ ). The results showed that SSd presented a significant inhibitory effect on cell proliferation simulated EGFR inhibitor or p38 inhibitor. So, a conclusion can be drawn based on the above findings that SSd inhibits cell growth via the EGFR/p38 MAPK signaling pathway.

\section{Discussion}

Currently available medical interventions for RCC, one of the most common malignant tumors in the world, are still relatively low in satisfaction in terms of the efficacy and safety. Surgical intervention can do some help to early stage RCC only, while patients with advanced tumor are still in urgent need for more promising approaches. Although studies have proved that SSd can inhibit cell growth and induce apoptosis in malignancies such as liver cancer, prostate cancer and thyroid carcinoma $[8,23,24]$, its effect in RCC remains unclear. Our study is the first to explore the role and mechanism of SSd in RCC treatment which has been proved in this research to be able to inhibit RCC cell growth in vitro.

It is well known that abnormal cell proliferation plays a major part in tumorigenesis. In this study, the inhibitory effect of SSd on cell proliferation was found in two RCC cell lines--769-P and 786-O by MTT assay. Consistent with the findings of R-Y LIU's studies, SSd, even at a relatively low dose 
A SSd

769-P
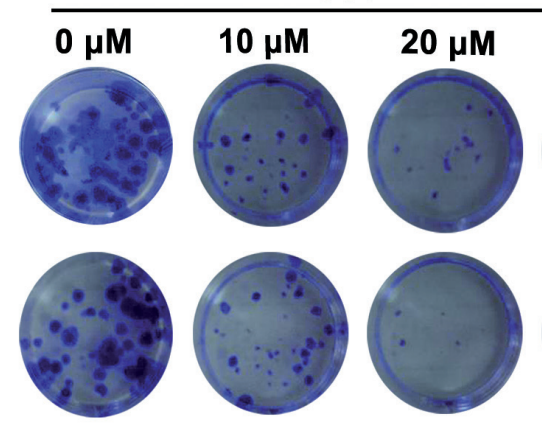

$30 \mu \mathrm{M}$

786-0

B

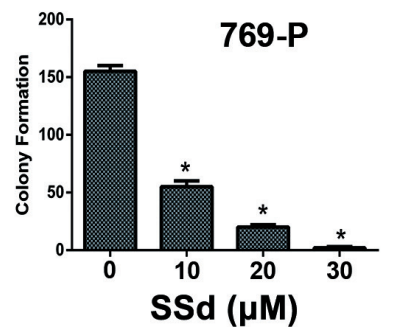

C

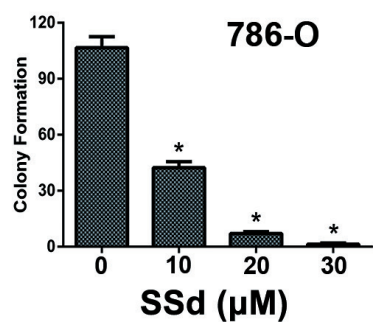

Figure 2. The inhibitory effect of SSd on colony formation of RCC cells. (A) Colony formation assay (24-well). 769-P and 786-O cells were respectively seeded into 24 -well plates, 100 cells per well, and cultured after treatment respectively with different doses of SSd $(10,20$ and $30 \mu \mathrm{M})$ for 14 days for crystal violet staining and colony number counting. (B and $\mathrm{C}$ ) Colony quantification. ${ }^{\star} \mathrm{P}<0.05$ represents a statistically significant difference.

$(20 \mu \mathrm{M})$, showed an effective inhibition on RCC cell growth in our study, suggesting a feature of low toxicity and good safety in the future clinical use, which is subject to further verification in subsequent clinical trials [9].

Cell cycle checkpoints are key factors to control cell cycle and influence the cell proliferation, and those at the G1-S transition and at the G2-M transition have great role in detecting and correcting DNA damage [25]. p53 serves as the most important tumor suppressor protein to regulate the cell cycle checkpoints, particularly those at the G1/S transition which can respond to DNA damage and induce G0/G1 arrest [2628]. Apoptosis induction achieved through the death receptor pathway and the intrinsic or mitochondrial pathway is a vital mechanism in organ development by which the homeostasis is sustained [29]. As abnormal cell proliferation mostly accounts for the pathogenesis for tumor formation, interventions involving with inducing apoptosis and cell cycle arrest are considered to be potentially effective solutions in anti-tumor treatment. Ya-Ling Hsu revealed that the proliferation inhibitory effect and apoptosis promoting activity of SSd were obtained through inducing p53 and the Fas/FasL apoptotic system in human non-small cell lung cancer A549 cell lines. Cell apoptosis and cell cycle arrest induced by SSd in human liver cancer cells was also demonstrated in another study which associated the above effects with p53 stimulation as well as the further induction of Bax and p21 expression [9]. In our study, G0/G1 cell cycle arrest was successfully induced after SSd treatment, with an
A
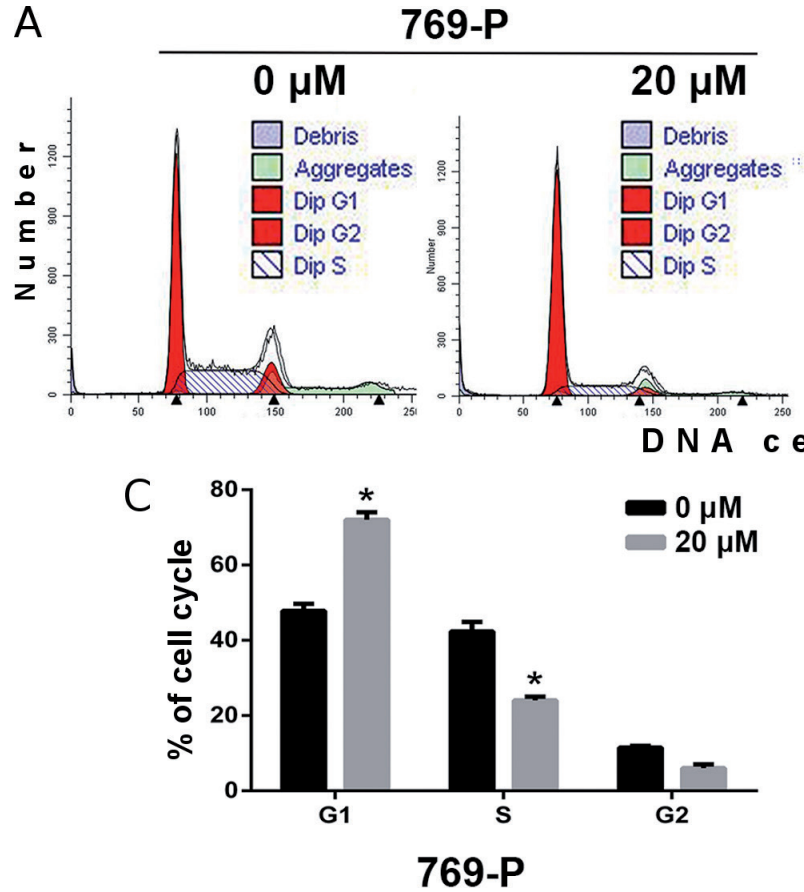

B
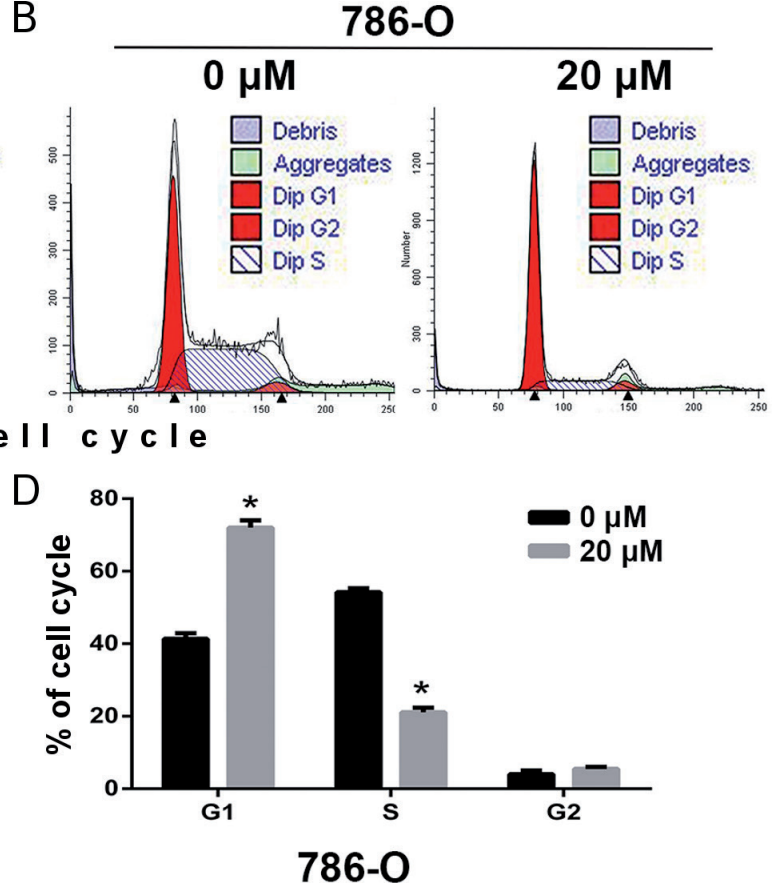

Figure 3. Activity of SSd on cell cycle arrest induction in RCC cell lines. (A) (B) Cell cycle analysis. 769-P and 786-O cells were treated respectively with indicated doses of SSd, fixed and stained with PI, and then analyzed by DNA contents using FACS. (C) (D) Comparison of cell quantification between different stages of the cell cycle. ${ }^{\star} \mathrm{P}<0.05$ represents a statistically significant difference. 
increased expression of p53 protein, indicating that SSd can inhibit the proliferation of RCC cells.

The molecular mechanism of SSd-induced apoptosis and cell cycle arrest in RCC was also investigated in this study based on the previous finding that EGFR/p38 signaling pathways are those involving in cell growth, differentiation and migration, and are activated in RCC cells. In this study, a down-regulation suggesting apoptosis induction was detected in some cell growth related genes as well as phospho-p38 and its downstream c-Fos, with the exception of phospho-ERK which, however, showed an increase after SSd treatment, which might be explained by its activity on inducing ER stress [30]. The proteins upstream of p38 with cell growth regulation activity such as $\mathrm{p}$-MEK and p-EGFR were also tested, leading to a conclusion that SSd may in- hibit cell growth through the EGFR/p38 signaling pathway. The protein lever of EGFR, MEK, p38, as p-EGFR, p-MEK, p-p38, deceased with treatment of SSd, so we can guess that SSd not only decrease phosph level, but also degrade total protein level. EGFR inhibitor AG1478 and p38 inhibitor SB203580 were utilized in the study as positive controls to make a comparison between them and SSd on their cell proliferation inhibitory effects. The results of these tests showed a strong support to the hypothesis that the EGFR/p38 signaling pathway is closely associated with the cell growth inhibitory effect of SSd in RCC cell lines.

In conclusion, the present study demonstrates for the first time that SSd has proliferation inhibitory effects in RCC cells. And its anti-proliferative activity is very likely to be mediated by apoptosis and cell cycle arrest induction via the

\section{A}

SSd
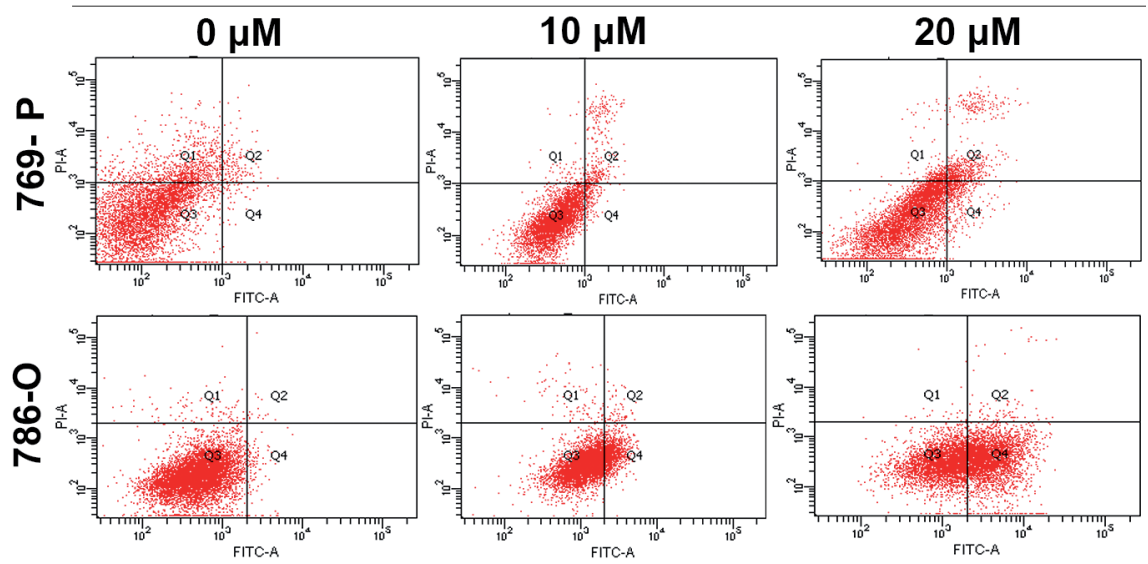

B
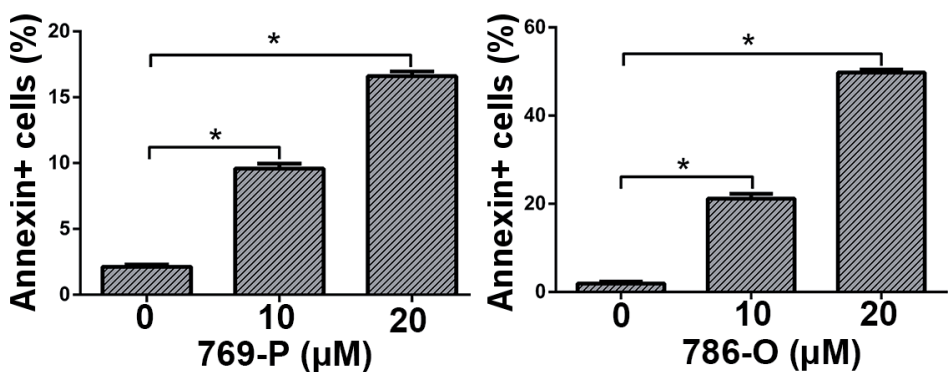

C

769-P SSd

786-O SSd

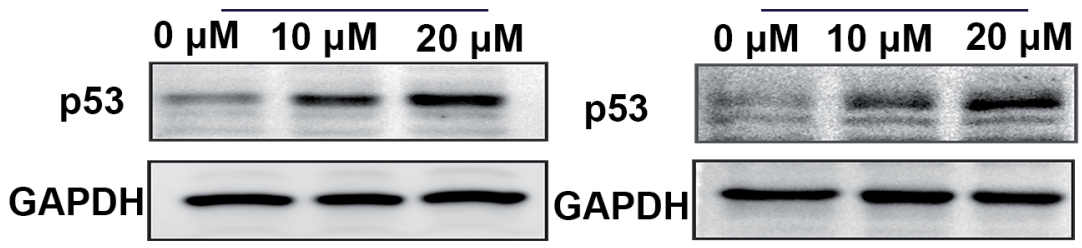

Figure 4. The effect of SSd on apoptosis induction in RCC cell lines. (A) 769-P and 786-O cells were treated respectively with 10 and $20 \mu \mathrm{M}$ of SSd for $48 \mathrm{~h}$, and then stained with Annexin V and PI for FACS analysis. (B) Comparison of Annexin V positive cell quantification between different dose groups. (C) p53 (1:1,000) analysis using western blotting assay after a similar SSd treatment procedure. ${ }^{\star} \mathrm{P}<0.05$ represents a statistically significant difference. 
strong inhibitory activation on EGFR/p38 pathways as well as up-regulated expression of p53 protein [31]. The results of this study suggest that SSd could be considered as a promising candidate for further research and clinical use in RCC patients. Certainly, more pre-clinical studies are needed to verify the efficacy and safety of SSd treatment in RCC models, which could serve as the basis for the design of clinical trials in RCC patients.

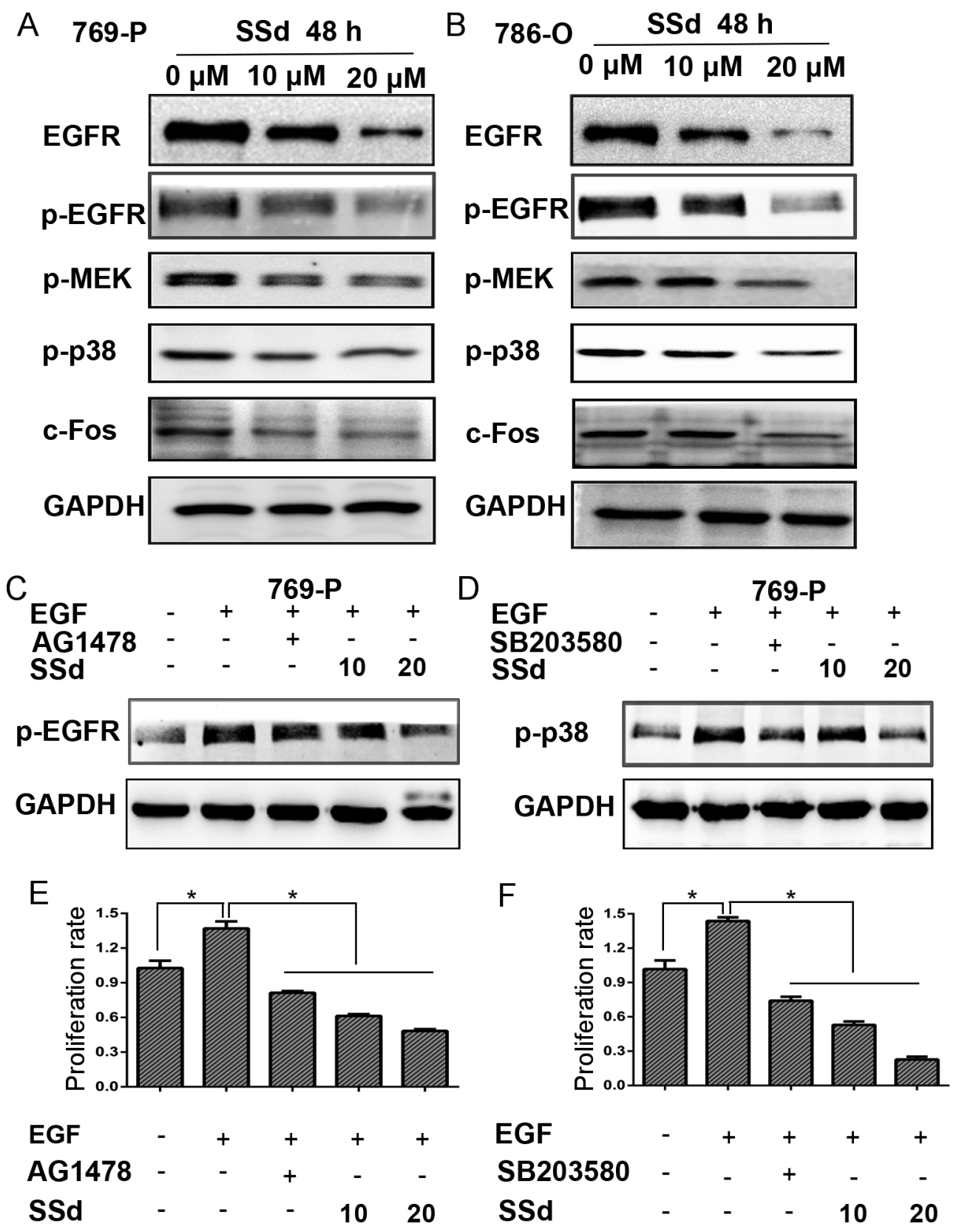

Figure 5. SSd performs its cell growth inhibitory function via the EGFR/p38 MAPK signaling pathway. (A and B) SSd-treated 769-P and 786-O cells were analyzed to determine EGFR, p-EGFR, MEK, p-MEK, p38, p-p38, and c-Fos by western blotting, with $\beta$-Actin, GAPDH used as a loading control. (C and D) 769-P cells were treated respectively with different doses of SSd for $48 \mathrm{~h}$, with AG1478 (EGFR inhibitor) or SB203580 (p38 inhibitor) as the positive control; before cell collection, the cells were treated with EGF for 30 min to activate p-EGFR or phospho-p38, which was determined using western blotting. ( $\mathrm{E}$ and F) Cells were seeded in 24 -well plates, and then treated with SSd $(2 \mathrm{~h})$ and EGF (48 h) for cell proliferation rate determination by MTT assay, with AG1478 or SB203580 used as the positive control. ${ }^{\star} \mathrm{P}<0.05$ represents a statistically significant difference. 
Ackowledgements: This work was supported by National Natural Science Foundation of China (No.81502577), Southern Medical University Foundation (No.PY2014N043), and Nanfang Hospital Foundation (No.2014C007).

\section{References}

[1] BROOKMAN-MAY S, BURGER M, WIELAND WF, ROSSLER W, MAY M et al. Vaccination therapy in renal cell carcinoma: current position and future options in metastatic and localized disease. Expert Rev Vaccines 2011; 10: 837-852. https://doi.org/10.1586/erv.11.64

[2] ESCUDIER B, EISEN T, PORTA C, PATARD JJ, KHOO V et al. Renal cell carcinoma: ESMO Clinical Practice Guidelines for diagnosis, treatment and follow-up. Ann Oncol 2012; 23 Suppl 7: vii65-71. https://doi.org/10.1093/annonc/mds227

[3] CHIONG E, TAY MH, TAN MH, KUMAR S, SIM HG et al. Management of kidney cancer in Asia: resource-stratified guidelines from the Asian Oncology Summit 2012. Lancet Oncol 2012; 13: e482-491. https://doi.org/10.1016/S14702045(12)70433-3

[4] PARKIN DM, BRAY F, FERLAY J, PISANI P. Global cancer statistics, 2002. CA Cancer J Clin 2005; 55: 74-108. https:// doi.org/10.3322/canjclin.55.2.74

[5] WONG VK, ZHANG MM, ZHOU H, LAM KY, CHAN PL et al. Saikosaponin-d Enhances the Anticancer Potency of TNF-alpha via Overcoming Its Undesirable Response of Activating NF-Kappa B Signalling in Cancer Cells. Evid Based Complement Alternat Med 2013; 2013: 745295. https://doi. org/10.1155/2013/745295

[6] DANG SS, WANG BF, CHENG YA, SONG P, LIU ZG et al. Inhibitory effects of saikosaponin-d on CCl4-induced hepatic fibrogenesis in rats. World J Gastroenterol 2007; 13: 557-563. https://doi.org/10.3748/wjg.v13.i4.557

[7] CHIANG LC, NG LT, LIU LT, SHIEH DE, LIN CC. Cytotoxicity and anti-hepatitis B virus activities of saikosaponins from Bupleurum species. Planta Med 2003; 69: 705-709. https:// doi.org/10.1055/s-2003-42797

[8] LIU RY, LI JP. Saikosaponin-d inhibits proliferation of human undifferentiated thyroid carcinoma cells through induction of apoptosis and cell cycle arrest. Eur Rev Med Pharmacol Sci 2014; 18: 2435-2443.

[9] HSU YL, KUO PL, CHIANG LC, LIN CC. Involvement of p53, nuclear factor kappaB and Fas/Fas ligand in induction of apoptosis and cell cycle arrest by saikosaponin d in human hepatoma cell lines. Cancer Lett 2004; 213: 213-221. https:// doi.org/10.1016/j.canlet.2004.03.044

[10] WANG BF, DAI ZJ, WANG XJ, BAI MH, LIN S et al. Saikosaponin-d increases the radiosensitivity of smmc-7721 hepatocellular carcinoma cells by adjusting the g0/g1 and $\mathrm{g} 2 / \mathrm{m}$ checkpoints of the cell cycle. BMC Complement Altern Med 2013; 13: 263. https://doi.org/10.1186/1472-6882-13$\underline{263}$

[11] SONG W, DANG Q, XU D, CHEN Y, ZHU G et al. Kaempferol induces cell cycle arrest and apoptosis in renal cell carcinoma through EGFR/p38 signaling. Oncol Rep 2014; 31: $1350-1356$.
[12] SHIN MS, SHINGHIRUNNUSORN P, SUGISHIMA Y, NISHIMURA M, SUZUKI $S$ et al. Cross interference with TNF-alpha-induced TAK1 activation via EGFR-mediated p38 phosphorylation of TAK1-binding protein 1. Biochim Biophys Acta 2009; 1793: 1156-1164. https://doi.org/10.1016/j. bbamcr.2009.04.005

[13] HYNES NE, LANE HA. ERBB receptors and cancer: the complexity of targeted inhibitors. Nat Rev Cancer 2005; 5: 341-354. https://doi.org/10.1038/nrc1609

[14] AMARE KADAM PS, VARGHESE C, BHARDE SH, NARASIMHAMOORTHY NK, DESAI S et al. Proliferating cell nuclear antigen and epidermal growth factor receptor (EGFr) status in renal cell carcinoma patients with polysomy of chromosome 7. Cancer Genet Cytogenet 2001; 125: 139-146. https://doi.org/10.1016/S0165-4608(00)00375-7

[15] MOCH H, SAUTER G, GASSER TC, BUBENDORF L, RICHTER J et al. EGF-r gene copy number changes in renal cell carcinoma detected by fluorescence in situ hybridization. J Pathol 1998; 184: 424-429. https://doi. org/10.1002/(SICI) 1096-9896(199804)184:4<424::AIDPATH1223>3.0.CO;2-A

[16] DORDEVIC G, MATUSAN ILIJAS K, HADZISEJDIC I, MARICIC A, GRAHOVAC B et al. EGFR protein overexpression correlates with chromosome 7 polysomy and poor prognostic parameters in clear cell renal cell carcinoma. J Biomed Sci 2012; 19: 40. https://doi.org/10.1186/1423-0127-19-40

[17] CHEN MF, HUANG CC, LIU PS, CHEN CH, SHIU LY. Saikosaponin a and saikosaponin $\mathrm{d}$ inhibit proliferation and migratory activity of rat HSC-T6 cells. J Med Food 2013; 16: 793-800. https://doi.org/10.1089/jmf.2013.2762

[18] LI Y, WANG K, ZOU QY, MAGNESS RR, ZHENG J. 2,3,7,8-Tetrachlorodibenzo-p-dioxin differentially suppresses angiogenic responses in human placental vein and artery endothelial cells. Toxicology 2015; 336: 70-78. https://doi. org/10.1016/j.tox.2015.08.003

[19] WANG K, LI Y, JIANG YZ, DAI CF, PATANKAR MS et al. An endogenous aryl hydrocarbon receptor ligand inhibits proliferation and migration of human ovarian cancer cells. Cancer Lett 2013; 340: 63-71. https://doi.org/10.1016/j.can$\underline{\text { let.2013.06.026 }}$

[20] MARSHALL CJ. Specificity of receptor tyrosine kinase signaling: transient versus sustained extracellular signalregulated kinase activation. Cell 1995; 80: 179-185. https:// doi.org/10.1016/0092-8674(95)90401-8

[21] HAN Y, CADAY CG, NANDA A, CAVENEE WK, HUANG HJ. Tyrphostin AG 1478 preferentially inhibits human glioma cells expressing truncated rather than wild-type epidermal growth factor receptors. Cancer Res 1996; 56: 3859-3861.

[22] CLERK A, SUGDEN PH. The p38-MAPK inhibitor, SB203580, inhibits cardiac stress-activated protein kinases/ c-Jun N-terminal kinases (SAPKs/JNKs). FEBS Lett 1998; 426: 93-96. https://doi.org/10.1016/S0014-5793(98)00324$\underline{\mathrm{X}}$

[23] YAO M, YANG J, CAO L, ZHANG L, QU S et al. Saikosaponind inhibits proliferation of DU145 human prostate cancer cells by inducing apoptosis and arresting the cell cycle at G0/ G1 phase. Mol Med Rep 2014; 10: 365-372. 
[24] HE S, LU G, HOU H, ZHAO Z, ZHU Z et al. Saikosaponind suppresses the expression of cyclooxygenase 2 through the phosphosignal transducer and activator of transcription 3/ hypoxiainducible factorlalpha pathway in hepatocellular carcinoma cells. Mol Med Rep 2014; 10: 2556-2562.

[25] GUO LD, CHEN XJ, HU YH, YU ZJ, WANG D et al. Curcumin inhibits proliferation and induces apoptosis of human colorectal cancer cells by activating the mitochondria apoptotic pathway. Phytother Res 2013; 27: 422-430. https://doi. org/10.1002/ptr.4731

[26] HARTWELL LH, KASTAN MB. Cell cycle control and cancer. Science 1994; 266: 1821-1828. https://doi.org/10.1126/ $\underline{\text { science. } 7997877}$

[27] KASTAN MB, ONYEKWERE O, SIDRANSKY D, VOGELSTEIN B, CRAIG RW. Participation of p53 protein in the cellular response to DNA damage. Cancer Res 1991; 51: 6304-6311.
[28] KUERBITZ SJ, PLUNKETT BS, WALSH WV, KASTAN MB. Wild-type p53 is a cell cycle checkpoint determinant following irradiation. Proc Natl Acad Sci U S A 1992; 89: 7491-7495. https://doi.org/10.1073/pnas.89.16.7491

[29] CHALAH A, KHOSRAVI-FAR R. The mitochondrial death pathway. Adv Exp Med Biol 2008; 615: 25-45. https://doi. org/10.1007/978-1-4020-6554-5 3

[30] ZHANG LJ, CHEN S, WU P, HU CS, THORNE RF et al. Inhibition of MEK blocks GRP78 up-regulation and enhances apoptosis induced by ER stress in gastric cancer cells. Cancer Lett 2009; 274: 40-46. https://doi.org/10.1016/j.canlet.2008.08.030

[31] LI L, GAO Y, ZHANG L, ZENG J, HE D et al. Silibinin inhibits cell growth and induces apoptosis by caspase activation, down-regulating survivin and blocking EGFR-ERK activation in renal cell carcinoma. Cancer Lett 2008; 272: 61-69. https:// doi.org/10.1016/j.canlet.2008.06.033 